\title{
Endoscopic management of gastrointestinal motility disorders - part 2: European Society of Gastrointestinal Endoscopy (ESGE) Guideline
}

Authors

Bas L. A. M. Weusten ${ }^{1,2}$, Maximilien Barret ${ }^{3}$, Albert J. Bredenoord ${ }^{4}$, Pietro Familiari' ${ }^{5}$, Jean-Michel Gonzalez ${ }^{6}$, Jeanin E. van Hooft ${ }^{4}$, Vicente Lorenzo-Zúñiga ${ }^{7}$, Hubert Louis ${ }^{8}$, Jan Martinek ${ }^{9}$, Suzanne van Meer ${ }^{1,2}$, Helmut Neumann ${ }^{10}$, Daniel Pohl ${ }^{11}$, Frederic Prat ${ }^{3}$, Daniel von Renteln ${ }^{12}$, Edoardo Savarino ${ }^{13}$, Rami Sweis ${ }^{14}$, Jan Tack ${ }^{15}$, Radu Tutuian ${ }^{16}$, Sauid Ishaq $^{17}$

Institutions

1 Department of Gastroenterology and Hepatology, University Medical Center Utrecht, Utrecht University, The Netherlands

2 Department of Gastroenterology and Hepatology, St Antonius Hospital, Nieuwegein, The Netherlands

3 Department of Gastroenterology and Digestive Oncology, Cochin Hospital, Assistance PubliqueHopitaux de Paris and University of Paris, France

4 Department of Gastroenterology, Amsterdam UMC, Location AMC, Amsterdam, The Netherlands

5 Digestive Endoscopy Unit, Università Cattolica del Sacro Cuore, Fondazione Policlinico Universitario A. Gemelli, IRCCS, Rome, Italy

6 Department of Gastroenterology, Hôpital Nord, Marseille, France

7 Endoscopy Unit, University Hospital La Fe, Valencia, Spain

8 Department of Gastroenterology, Hepatopancreatology and Digestive Oncology, Erasme Hospital, Université Libre de Bruxelles, Brussels, Belgium

9 Department of Hepatogastroenterology, Institute for Clinical and Experimental Medicine, Prague, Czech Republic

10 Department of Medicine I, University Medical Center Mainz, Mainz, Germany

11 Department of Gastroenterology, University Hospital Zurich, Zurich, Switzerland

12 Division of Gastroenterology, Montréal University Hospital (CHUM), Montréal, Canada

13 Department of Surgery, Oncology and Gastroenterology, DiSCOG, University of Padova, Padova, Italy

14 Department of Gastroenterology, University College London Hospital, London, UK
15 Department of Gastroenterology, University Hospitals Leuven, Leuven, Belgium

16 Department of Gastroenterology, University Clinic for Visceral Surgery and Medicine, Bern University Hospital, Bern, Switzerland

17 Department of Gastroenterology, Dudley Group NHS Foundation Trust and Birmingham City University, Birmingham, UK

Bibliography

DOI https://doi.org/10.1055/a-1171-3174

Published online: 25.5.2020 | Endoscopy 2020; 52: 600-614

(c) Georg Thieme Verlag KG Stuttgart · New York

ISSN 0013-726X

Corresponding author

Bas L.A.M. Weusten, MD, PhD, Dept of Gastroenterology and Hepatology, University Medical Center Utrecht, Internal mail no F02.618, P.O. Box 85500, 3508 GA UTRECHT, The Netherlands b.l.a.weusten@umcutrecht.nl

丹 Appendix 1s-3s

Online content viewable at:

https://doi.org/10.1055/a-1171-3174

\section{MAIN RECOMMENDATIONS}

ESGE suggests flexible endoscopic treatment over open surgical treatment as first-line therapy for patients with a symptomatic Zenker's diverticulum of any size.

Weak recommendation, low quality of evidence, level of agreement $100 \%$.

ESGE recommends that emerging treatments for Zenker's diverticulum, such as Zenker's peroral endoscopic myotomy (Z-POEM) and tunneling, be considered as experimental; 
these treatments should be offered in a research setting only.

Strong recommendation, low quality of evidence, level of agreement $100 \%$.

ESGE recommends against the widespread clinical use of transoral incisionless fundoplication (TIF) as an alternative to proton pump inhibitor (PPI) therapy or antireflux surgery in the treatment of gastroesophageal reflux disease (GERD), because of the lack of data on the long-term outcomes, the inferiority of TIF to fundoplication, and its modest efficacy in only highly selected patients. TIF may have a role for patients with mild GERD who are not willing to take PPIs or undergo antireflux surgery.

Strong recommendation, moderate quality of evidence, level of agreement $92.8 \%$.

ESGE recommends against the use of the Medigus ultrasonic surgical endostapler (MUSE) in clinical practice because of insufficient data showing its effectiveness and safety in patients with GERD. MUSE should be used in clinical trials only.

Strong recommendation, low quality evidence, level of agreement $100 \%$.

ESGE recommends against the use of antireflux mucosectomy (ARMS) in routine clinical practice in the treatment of GERD because of the lack of data and its potential complications.
Strong recommendation, low quality evidence, level of agreement $100 \%$.

ESGE recommends endoscopic cecostomy only after conservative management with medical therapies or retrograde lavage has failed.

Strong recommendation, low quality evidence, level of agreement $93.3 \%$.

ESGE recommends fixing the cecum to the abdominal wall at three points (using T-anchors, a double-needle suturing device, or laparoscopic fixation) to prevent leaks and infectious adverse events, whatever percutaneous endoscopic cecostomy method is used.

Strong recommendation, very low quality evidence, level of agreement $86.7 \%$.

ESGE recommends considering endoscopic decompression of the colon in patients with Ogilvie's syndrome that is not improving with conservative treatment.

Strong recommendation, low quality evidence, level of agreement $93.8 \%$.

ESGE recommends prompt endoscopic decompression if the cecal diameter is $>12 \mathrm{~cm}$ and if the Ogilvie's syndrome exists for a duration of longer than 4-6 days.

Strong recommendation, low quality evidence, level of agreement $87.5 \%$.

\section{SOURCE AND SCOPE}

This Guideline is an official statement of the European Society of Gastrointestinal Endoscopy (ESGE). It provides guidance on the endoscopic management of Zenker's diverticulum, gastroesophageal reflux disease, intractable constipation, and Ogilvie's syndrome. The Grading of Recommendations Assessment, Development, and Evaluation (GRADE) system was adopted to define the strength of recommendations and the quality of evidence.

\section{Introduction}

Therapeutic gastrointestinal (GI) endoscopy is rapidly evolving. Its role in the management of motility disorders of the digestive tract is increasing. The purpose of this Guideline is to provide guidance on various aspects of the endoscopic management of Gl motility disorders. This is the second of two parts of the guideline, and is dedicated to Zenker's diverticulum, gastroesophageal reflux disease (GERD), intractable constipation, and Ogilvie's syndrome. The first part, published as a separate manuscript, focused on achalasia and gastroparesis.

\section{Methodology}

The ESGE commissioned this Guideline (Guideline Committee chair, J.v.H.) and appointed a Guideline leader (B.W.); he identified six clinical conditions of abnormal Gl motility in which therapeutic endoscopy is one of the treatment possibilities: Zenker's diverticulum, achalasia, GERD, gastroparesis, intractable constipation, and Ogilvie's syndrome. These six areas were at a later stage agreed on by the Guideline committee members.

In March 2018, an email was sent out to several key opinion leaders in the field of therapeutic endoscopy to identify potential Guideline committee members. Individual ESGE members were informed about this Guideline and were asked to apply if they were interested in participating with this Guideline. Three individual members (V.L.-Z., H.L., and F.P.) were selected based on their expertise and scientific output. In addition, the European Society of Neurogastroenterology and Motility (ESNM) was approached for collaboration and scientific input. As a result, the ESNM appointed on request four Guideline committee members regarded as experts in the field of GI motility and therapy (D.P., E.S., J.T., and R.T.). Finally, a Guideline committee was formed comprising 18 members, and covering the six areas of this guideline. Six task forces were created, based on the six clinical conditions. Each task force had one or two task 


\begin{tabular}{|c|c|}
\hline \multicolumn{2}{|c|}{ ABBREVIATIONS } \\
\hline ARMS & antireflux mucosectomy \\
\hline CT & computed tomography \\
\hline EG] & esophagogastric junction \\
\hline EMR & endoscopic mucosal resection \\
\hline ESD & endoscopic submucosal dissection \\
\hline ESGE & $\begin{array}{l}\text { European Society of Gastrointestinal Endos- } \\
\text { copy }\end{array}$ \\
\hline ESNM & $\begin{array}{l}\text { European Society of Neurogastroenterology } \\
\text { and Motility }\end{array}$ \\
\hline GERD & gastroesophageal reflux disease \\
\hline GI & gastrointestinal \\
\hline G-POEM & gastric peroral endoscopic myotomy \\
\hline GRADE & $\begin{array}{l}\text { Grading of Recommendations Assessment, } \\
\text { Development, and Evaluation }\end{array}$ \\
\hline LAPEC & $\begin{array}{l}\text { laparoscopically assisted percutaneous } \\
\text { endoscopic cecostomy }\end{array}$ \\
\hline LES & lower esophageal sphincter \\
\hline PEG & polyethylene glycol \\
\hline POEM & peroral endoscopic myotomy \\
\hline PPI & proton pump inhibitor \\
\hline RCT & randomized controlled trial \\
\hline \multicolumn{2}{|c|}{ SB knife Jr. Stag Beetle knife Junior } \\
\hline TIF & transoral incisionless fundoplication \\
\hline UEG & United European Gastroenterology \\
\hline Z-POEM & Zenker's POEM \\
\hline
\end{tabular}

force leaders, and each group member was assigned to one or more task forces (Appendix 1s, see online-only Supplementary Material). The kick-off meeting for this Guideline was held during United European Gastroenterology (UEG) Week, on 21 October 2018, in Vienna.

During a teleconference in November 2018, clinical questions were formulated for the six clinical conditions. Subsequently, these clinical questions were translated into research questions (Appendix 2s). The questions followed the PICO format ( $P$, population in question; I, intervention; $C$, comparator; and $\mathrm{O}$, outcomes of interest) wherever appropriate. Subsequently, systematic literature searches were done using Medline, Embase, and Cochrane library.

Evidence levels and recommendation strengths were assessed using the Grading of Recommendations Assessment, Development and Evaluation (GRADE) system [1]. Further details on the methodology of ESGE guidelines have been reported elsewhere [2]. The results of data extraction are presented in Appendix 3s.

Available literature, draft recommendations, and strength of evidence were discussed during a face-to-face meeting with all group members at Schiphol Airport, Amsterdam on 12 April 2019.

In order to establish consensus-based recommendations, a modified Delphi process [3] was organized using an online voting platform (www.surveymonkey.com). Voting was based upon a five-point Likert scale (1, strongly disagree; 2, disagree;
3, neither disagree nor agree; 4, agree; 5, strongly agree). A recommendation was approved if $>75 \%$ of the members agreed (reflected by a Likert score of $4-5$ ). In total three iterations of the online voting process were needed to come to the final document.

In January 2020, a draft prepared by B.W. was sent to all group members. After the agreement of all group members had been obtained, the manuscript was reviewed by the ESGE Guideline Committee Chair (J.v.H.) and two external reviewers, and was sent for further comments to the ESGE national societies and individual members. After this, it was submitted to Endoscopy for publication.

\section{Zenker's diverticulum}

Zenker's diverticulum is a pulsion diverticulum that develops in an area of weakness of the posterior hypopharynx known as the Killian triangle (between the thyropharyngeus and cricopharyngeal muscle fibers of the inferior constrictor). It is a relatively uncommon condition, with an overall prevalence estimated to be between $0.01 \%$ and $0.11 \%$ in the American population; it occurs most frequently in men between their 7th and 8th decades $[4,5]$. Clinically, Zenker's diverticulum may manifest with symptoms such as dysphagia or regurgitation, and its associated complications [6].

The pathophysiology of Zenker's diverticulum is not fully understood, but the most widely proposed hypothesis is that a motor abnormity of the cricopharyngeus muscle creates a high pressure zone that facilitates herniation of the hypopharyngeal mucosa through the weak zone, the Killian triangle, resulting in diverticulum development $[7,8]$.

\subsection{Diagnosis of Zenker’s diverticulum}

\section{RECOMMENDATIONS}

ESGE recommends the use of barium swallow radiography with video fluoroscopy in the evaluation of patients with (suspected) Zenker's diverticulum.

Strong recommendation, very low quality of evidence, level of agreement $100 \%$.

ESGE suggests against the use of manometry as standard in the diagnostic work-up of patients with Zenker's diverticulum.

Weak recommendation, very low quality of evidence, level of agreement $100 \%$.

The diagnosis of Zenker's diverticulum is suspected on the basis of clinical symptoms and is confirmed by a barium swallow with video fluoroscopy. Although Zenker's diverticulum can be diagnosed by endoscopy, fluoroscopy is considered essential because it not only provides information on pouch size, but will also give "dynamic" information on regurgitation and aspiration. This is important in determining whether Zenker's diverticulum is the real cause of a patient's symptoms. 
Studies on the clinical value of manometry in Zenker's diverticulum are missing. The working group however recommends against the routine use of esophageal manometry in the workup of patients with (suspected) Zenker's diverticulum. In individual cases (e.g. in small Zenker's diverticulum), esophageal manometry can be used to rule out other causes of similar symptoms.

\subsection{Treatment options for Zenker's diverticulum}

\subsubsection{First-line treatment}

\section{RECOMMENDATION}

ESGE suggests flexible endoscopic treatment over open surgical treatment as first-line therapy for patients with a symptomatic Zenker's diverticulum of any size. Weak recommendation, low quality of evidence, level of agreement $100 \%$.

Treatment is indicated for symptomatic Zenker's diverticulum. Currently, there are three main treatment options for Zenker's diverticulum: open surgery (i.e. transcervical diverticulectomy, diverticulopexy with myotomy of the cricopharyngeus muscle, or diverticular inversion) $[9,10]$; rigid endoscopy (i.e. endoscopic stapling or $\mathrm{CO}_{2}$ laser treatment) [11, 12]; and flexible endoscopy. The key common intervention in all three options is division of the cricopharyngeus muscle that forms the septum between esophagus and the pouch. The goal of treatment is to reduce the size of the diverticulum and improve pharyngeal motor function, thus improving the symptoms of dysphagia and regurgitation.

Flexible endoscopic septum division involves the use of a flexible endoscope to carry out septal myotomy [13]. Various incision techniques have been described for cutting the septum that contains the cricopharyngeus muscle. The single-incision technique involves a midline incision of the cricopharyngeus muscle with the option of clipping the base [14]. The doubleincision technique allows a wider septum to be dissected. It involves creating two incisions that are $1 \mathrm{~cm}$ apart from each other and the septum in between is resected using a snare before the base is clipped [15].

There are no prospective comparative studies between surgery (by rigid endoscopic or open approach) and flexible endoscopic treatment. One large retrospective study by Shahawy et al. compared 36 patients treated by endoscopic septotomy with 31 patients treated by diverticulectomy and myotomy, and found dysphagia recurrence in $39 \%$ vs. $0 \%(P=0.001)$ at 2 months in the endoscopic vs. surgical treatment groups, respectively, with $13 \%$ vs. $31 \%$ complication rates after each treatment $(P=0.08)$ [16]. Two large systematic reviews and meta-analyses involving 3079 and 596 patients concluded that clinical success rates were significantly different, at $82 \%-87 \%$ with the endoscopic approach vs. $94 \%$ - $96 \%$ with the open surgical approach $[17,18]$. The complication rate was $7 \%-9 \%$ with the endoscopic treatment vs. $11 \%-15 \%$ with the open surgical approach.
Of note, most data from the literature mentioning endoscopic treatment for Zenker's diverticulum actually refer to laser or stapler septotomy using a rigid endoscope and performed by an ENT surgeon. This is important because rigid endoscopy is not always possible in elderly patients, with technical treatment failures of $6 \%-7 \%$ [17].

Most of the relevant data on the efficacy and safety of flexible endoscopic septotomy for the treatment of Zenker's diverticulum are summarized in the meta-analysis from Ishaq et al. [19]; however, for most of this data, there is no direct comparison to surgical treatment. In this work, including 813 patients, the pooled success rate of flexible endoscopic septotomy was $91 \%$, with an $11.3 \%$ adverse event rate and $11 \%$ recurrences. The limitations of surgery and rigid endoscopy, such as the need for general anesthesia and the high rate of intraoperative abandonment owing to restricted neck mobility in the elderly, combined with the seemingly higher complication rates of surgery with comparable success rates have led to our recommendation on the use of flexible endoscopic techniques as the firstline therapy for Zenker's diverticulum.

\subsubsection{Recurrent Zenker's diverticulum}

\section{RECOMMENDATION}

ESGE suggests treatment by flexible endoscopy for recurrent Zenker's diverticulum.

Weak recommendation, low quality of evidence, level of agreement $100 \%$.

Regardless of the treatment modality used, recurrence of Zenker's diverticulum is not uncommon. Predictors of symptom relapse that occurs within 48 months of endoscopic therapy include: pretreatment Zenker's diverticulum size $(\geq 50 \mathrm{~mm})$, post-treatment Zenker's diverticulum size $(\geq 10 \mathrm{~mm})$, and the length of the septotomy ( $\leq 25 \mathrm{~mm})$ [20].

Endoscopic management of recurrence after surgery or endoscopic stapling can be particularly challenging. No comparative studies have been carried out between surgery (endoscopic or open approaches) and flexible endoscopic treatment as therapy for pretreated patients with Zenker's diverticulum.

Two small retrospective studies involving 20 and 18 patients reported on the feasibility of rigid endoscopic treatment using a stapler for recurrent Zenker's diverticulum [21,22]. They reported a short-term clinical remission rate of $81 \%-90 \%$, with a $20 \%-28 \%$ complication rate after endoscopic therapy, and $5 \%$ recurrence rate. Antonello et al. reported on the feasibility, safety, and effectiveness of flexible endoscopic septotomy for recurrent Zenker's diverticulum in 25 patients, using a diverticuloscope and a septum incision or snare resection of the cricopharyngeus muscle, with similar outcomes when compared with data from 34 treatment-naïve patients treated within the same timeframe: the success, recurrence, and complication rates in naïve vs. recurrent patients were $84 \%$ vs. $82 \%, 24 \%$ vs. $15 \%$, and $8 \%$ vs. $8.8 \%$, respectively [15]. In their retrospective study using a needle-knife and diverticuloscope ( $n=134)$, Hub- 
erty et al. reported recurrence rates of $23.1 \%$; of those who underwent repeat treatment $(n=23), 78.3 \%$ achieved symptom remission after redo myotomy [14].

Therefore, although the data are limited, flexible endoscopic septotomy appears to perform equally well in recurrent Zenker's diverticulum and treatment-naïve Zenker's diverticulum.

\subsection{Flexible endoscopic treatment of Zenker's diverticulum}

\subsubsection{Use of a diverticuloscope}

\section{RECOMMENDATION}

ESGE suggests that it is left to the endoscopist's discretion whether or not to use a diverticuloscope when performing a flexible endoscopic septotomy for Zenker's diverticulum. Weak recommendation, low quality of evidence, level of agreement $100 \%$.

In one retrospective series, diverticuloscope-assisted treatment success was reported to be higher than cap-assisted treatment [23]. In a meta-analysis by Ishaq et al., however, overall use of a diverticuloscope had no impact on success or complications [19].

\subsubsection{Minimum size of Zenker's diverticulum}

\section{RECOMMENDATION}

ESGE suggests symptomatic Zenker's diverticula of any size are amenable to flexible endoscopic treatment, although the usefulness of a diverticuloscope remains uncertain for Zenker's diverticula $<2 \mathrm{~cm}$.

Weak recommendation, very low quality of evidence, level of agreement $100 \%$.

To our knowledge, no study has addressed the specific question of the minimum size of Zenker's diverticulum that is required for flexible endoscopic treatment. Published case series on flexible endoscopic septotomy have included patients with diverticula of mean size $20-50 \mathrm{~mm}$ [19]), with a possible optimal efficacy of endoscopic treatment for Zenker's diverticula between 30 and $50 \mathrm{~mm}$ in size [20].

Although most endoscopic studies have included patients with Zenker's diverticula measuring between 20 and $50 \mathrm{~mm}$, there is no minimum size for symptomatic Zenker's diverticula to be considered amenable to endoscopic treatment. If the size is below $20 \mathrm{~mm}$, however, the usefulness of a diverticuloscope is questionable.
3.3.3 Emerging endoscopic techniques for the treatment of Zenker's diverticulum

\section{RECOMMENDATION}

ESGE recommends that emerging treatments for Zenker's diverticulum, such as Zenker's peroral endoscopic myotomy (Z-POEM) and tunneling, be considered as experimental; these treatments should be offered in a research setting only.

Strong recommendation, low quality of evidence, level of agreement $100 \%$.

New, alternative strategies for treating Zenker's diverticulum by means of flexible endoscopy are emerging. For instance, tunneling techniques used to cut the lower esophageal sphincter (LES) in patients with achalasia (peroral endoscopic myotomy; POEM) or the pylorus in gastroparesis (gastric peroral endoscopic myotomy; G-POEM) have recently been applied to Zenker's diverticula as well. Yang et al. collected data on 75 patients treated by Zenker's POEM (Z-POEM) across 10 centers and found a technical success rate of $97 \%$, complication rate of $6.7 \%$ ( 1 bleed and 4 perforations - all managed conservatively), clinical success rate of $92 \%$, and observed only one recurrence after a median follow-up of 10 months [24]. To date, the reported studies on alternative endoscopic treatments for Zenker's diverticulum have mainly consisted of case reports and series that lack long-term follow-up data, with strong publication bias and possible underestimation of complication rates. More studies are needed to define their role in the management of Zenker's diverticulum.

\subsubsection{Use of $\mathrm{CO}_{2}$ during endoscopic Zenker's diverticulum treatment}

\section{RECOMMENDATION}

ESGE recommends routine use of $\mathrm{CO}_{2}$ in the endoscopic treatment of Zenker's diverticulum.

Strong recommendation, very low quality evidence, level of agreement $100 \%$.

Although there are no comparative data in Zenker's diverticulum, there is a sufficient body of evidence in a relatively comparable treatment modality, namely POEM for achalasia, that $\mathrm{CO}_{2}$ reduces the risk of subcutaneous emphysema. In Zenker's diverticulum, once the cricopharyngeus muscle has been dissected, the only posterior barrier to the superior mediastinum is the buccopharyngeal fascia. $\mathrm{CO}_{2}$ is reabsorbed more quickly than room air and its use reduces the risk of gas-related complications, such as pneumomediastinum and subcutaneous emphysema. 


\subsubsection{Use of prophylactic antibiotics}

\section{RECOMMENDATION}

ESGE does not recommend routine administration of prophylactic antibiotics before or after endoscopic septotomy for Zenker's diverticulum.

Strong recommendation, low quality of evidence, level of agreement $92.9 \%$.

In a meta-analysis by Ishaq et al., 7/20 studies on conventional endoscopic treatment of Zenker's diverticulum used prophylactic antibiotics. Meta-regression analysis for overall safety showed that prophylactic administration of antibiotics was not associated with a reduction in complications [19].

\subsubsection{Extent of myotomy}

\section{RECOMMENDATION}

ESGE recommends performing a complete myotomy of the cricopharyngeus muscle when performing endoscopic septotomy.

Strong recommendation, low quality of evidence, level of agreement $93.8 \%$.

All expert endoscopists involved in the treatment of Zenker's diverticulum by flexible endoscopy strongly advocate a full transection of the cricopharyngeus muscle. Direct evidence from the literature to support this is lacking; however, from a pathophysiological standpoint, a full myotomy of the cricopharyngeus muscle is essential to prevent the recurrence of symptoms. The value of cutting deeper is controversial.

Costamagna et al. reported failure at 6 months if the length of the septotomy was $<2.5 \mathrm{~cm}$ or the pretreatment pouch was $>5 \mathrm{~cm}$, and failure at 48 months if the septotomy length was $<2.5 \mathrm{~cm}$ or the post-procedure Zenker's diverticulum size $>10 \mathrm{~cm}$ [20]. However, the authors' measurement of septotomy length is debatable as they measured pouch size before and after treatment using a marked catheter. They extrapolated that the pouch size is equal to the septum length, but there is no peer evidence to support this assumption.

\subsubsection{Use of clips at the base of the septotomy}

\section{RECOMMENDATION}

ESGE suggests that the decision to deploy clips at the base of the septotomy should be dictated by endoscopist practice or clinical need (bleeding or suspected perforation).

Weak recommendation, low quality of evidence, level of agreement $100 \%$.
Clips are widely used at the base of the septotomy by the majority of endoscopists, despite there being no evidence of their impact on adverse events, such as bleeding or perforation. In a recent meta-analysis by Ishaq et al., six studies deployed clips during the procedure but meta-regression analysis showed this had no impact on adverse events, such as perforation [19].

\subsubsection{Endoscopic knives and electrocoagulation settings}

Almost every kind of endoscopic submucosal dissection (ESD) device has been used to treat Zenker's diverticulum. Early work was done by Ishioka with the needle-knife papillotome [25]. The advantages of the needle-knife include its low cost and easy availability, but its disadvantages include downward cutting, which is linked with complications.

The HookKnife (Olympus, Tokyo, Japan) was originally designed for ESD. The tip of the knife is bent at a right angle; with the rotatable "hook" measuring $1.3 \mathrm{~mm}$ and the arm measuring $4.5 \mathrm{~mm}$. This design enables the cricopharyngeal muscle fibers to be isolated, pulled upwards, and then cut. Theoretically, the upwards pull of the septal fibers minimizes perforation risk. Repici et al. reported a complication rate of $6.3 \%$ and overall success rate of $90.6 \%$ for HookKnife myotomy [26]. Similar findings were presented by Rouquette et al. who showed overall success rates of $91.7 \%$, complication rates of $8.4 \%$, and recurrence in $12.5 \%$ of patients [27].

The Stag Beetle knife Junior (SB knife Jr., Sumitomo Bakelite Co., Tokyo, Japan) is a scissor-shaped cutting tool that can be used to divide the septum and is often used with a diverticuloscope or cap. Both blades of the SB knife Jr. are insulated externally. It has two practical advantages over other cutting devices. First, the SB knife Jr. allows an incision from the apex to the base of the septum but with a scissor-like movement, which pulls the muscle fibers towards the endoscope while cutting. In addition, the $360^{\circ}$ rotational ability increases therapeutic precision and prevents unwanted deep incisions that may lead to perforation. In a retrospective study of 31 patients undergoing SB knife septal myotomy, Battaglia et al. described a median procedure time of only 14 minutes, with $83.9 \%$ of patients in remission from symptoms after a median follow-up of 7 months [28]. The efficacy and safety data were replicated in 52 patients by Goelder et al., who reported a low recurrence rate of $9.6 \%$ over 6 months, without the occurrence of perforation or mediastinitis [29].

The settings for the electrosurgical generators vary between the different brands and models and can be different for different devices. Therefore, specific settings for the electrosurgical generator being used should be requested from the manufacturer.

\subsubsection{Post-procedural care}

No specific recommendations regarding the postoperative care of patients can be deduced from the analysis of the current literature. However, after the procedure, patients should be carefully monitored to recognize possible complications. Patients are routinely kept nil per os for 24 hours. In many published series, patients were allowed liquid diet the next day if their 
course was unremarkable. There is no evidence to support a contrast study being a prerequisite to resume oral intake unless a perforation is suspected.

\section{Gastroesophageal reflux disease}

GERD is a common condition that affects approximately $8.8 \%$ $25.9 \%$ of European adults [30]. Medical therapy using proton pump inhibitors (PPIs) and surgical treatment by means of fundoplication are both proven to be effective. Some patients are either reluctant to use chronic medication or are allergic to PPIs, but do not want to undergo a surgical solution. In addition, chronic PPI use imposes significant costs.

In the past, several endoscopic treatment modalities have been evaluated, but most of these were finally withdrawn from the market owing to lack of efficacy or major side effects [31 37]. Nowadays, several new endoscopic modalities are on the market or are being evaluated.

\subsection{Transoral incisionless fundoplication (TIF)}

\section{RECOMMENDATION}

ESGE recommends against the widespread clinical use of transoral incisionless fundoplication (TIF) as an alternative to PPI therapy or antireflux surgery in the treatment of GERD, because of the lack of data on the long-term outcomes, the inferiority of TIF to fundoplication, and its modest efficacy in only highly selected patients. TIF may have a role for patients with mild GERD who are not willing to take PPIs or undergo antireflux surgery.

Strong recommendation, moderate quality of evidence, level of agreement $92.8 \%$.

Transoral incisionless fundoplication (TIF) is performed with an endoscopic suturing device using T-fasteners and aims to create a gastroplication that reinforces the antireflux barrier. TIF has been evaluated in five randomized controlled trials (RCTs) in patients mostly with moderate GERD, excluding those with large hiatal hernias $(>2 \mathrm{~cm})$, Los Angeles grade $C$ or D esophagitis, or Barrett's esophagus [38-42]. TIF was evaluated after 6 months of follow-up and compared with a sham procedure [42], a sham procedure and PPI therapy [41], or in an unblinded manner with PPI therapy [38-40]. A meta-analysis showed that a clinical response, defined by an improvement of at least $50 \%$ in the GERD health-related quality of life (GERDHRQL) scores or remission of heartburn and regurgitation, was observed in $66 \%$ of patients treated with TIF and $30 \%$ of the control groups [43]. Objective measurement of reflux showed a limited decrease in esophageal acid exposure in patients treated with TIF, a similar level of decrease to that observed in patients taking PPIs.

No long-term data are available from RCTs, but results from uncontrolled studies show decreased effectiveness over time, with PPI cessation rates ranging from $70 \%$ at 6 months to $34 \%$ at 5 years, which suggests the procedure has a short-term ben- efit in two-thirds of patients. Severe adverse events, including esophageal perforation and bleeding, have been reported in $2.4 \%$ of patients [43]. TIF was also compared to Nissen fundoplication in a prospective open study [44]. Objective and symptomatic evaluation of reflux showed superiority of the surgical fundoplication.

To conclude, TIF may improve GERD symptoms in the short term, but long-term control of reflux is not achieved in the majority of patients with well-characterized and uncomplicated GERD. The exact positioning of TIF in the armamentarium remains unclear: it might offer some symptomatic relief for patients who are intolerant to PPIs, not willing to take PPIs, or for those who have persistent regurgitation on PPIs but are reluctant to undergo antireflux surgery.

\subsection{Medigus ultrasonic surgical endostapler (MUSE)}

\section{RECOMMENDATION}

ESGE recommends against the use of the Medigus ultrasonic surgical endostapler (MUSE) in clinical practice because of insufficient data showing its effectiveness and safety in patients with GERD. MUSE should be used in clinical trials only.

Strong recommendation, low quality evidence, level of agreement $100 \%$.

The Medigus ultrasonic surgical endostapler (MUSE; Medigus, Omer, Israel) is a system that integrates flexible videoendoscopy with an ultrasonic range finder and a surgical stapler. At the center of the endoscope is a rigid section of approximately $66 \mathrm{~mm}$ that holds a cartridge containing five 4.8-mm standard "B"-shaped titanium surgical staples. The tip of the endoscope contains an anvil for the staples as well as two small screws. An ultrasonic range finder measures the distance between an ultrasonic mirror in the cartridge and the tip of the endoscope.

Currently, the MUSE device has been evaluated in a prospective multicenter trial including 66 patients with a short-term follow-up period [45]. After 6 months, the GERD-HRQL score improved by more than $50 \%$ while off PPI therapy in $73 \%$ of patients (95\% confidence interval [CI] 60\%-83\%) and 42 patients $(64.6 \%)$ were no longer using daily PPI medication. Two patients suffered from severe complications (empyema in one, hemorrhage in the other). The 4-year follow-up data were reported in 37 of the initial 66 patients [46]. Both the GERDHRQL and percentage of patients off PPIs had decreased slightly but significantly over time; however, they remained significantly better than at baseline.

Danalioglu et al. compared the results of the MUSE in 11 patients with laparoscopic fundoplication in 16 patients [47]. Patients however were not randomized, and a hiatal hernia of $>3 \mathrm{~cm}$ was an exclusion criterion for MUSE only. In this small retrospective study, laparoscopic fundoplication appeared to be more effective after a 6-month follow-up period, and one 
severe complication (esophageal perforation) was seen in the MUSE group.

Overall, data on the safety and efficacy of MUSE in the treatment of GERD are scarce and sham-controlled trials are lacking, as are studies randomizing patients between MUSE and laparoscopic fundoplication. ESGE therefore recommends against the use of MUSE outside of the context of clinical trials.

\subsection{Radiofrequency energy application to the LES (Stretta)}

\section{RECOMMENDATION}

ESGE suggests that Stretta can be considered in selective patients only, for the sake of symptom relief and in the absence of erosive esophagitis and a hiatal hernia. Weak recommendation, moderate level of evidence, level of agreement $92.9 \%$.

Radiofrequency energy application to the LES (Stretta; Restech, Houston, Texas, USA) is an endoscopically-guided method in which radiofrequency current is conducted by a series of radially arranged needles positioned over the esophagogastric junction (EGJ). Although the exact mechanism by which Stretta opposes further gastroesophageal reflux is still unclear, the technique is supposed to induce inflammatory changes that result in submucosal fibrosis, with a subsequent increase in LES pressure and/or decrease in LES compliance. Stretta is not recommended in patients with erosive esophagitis or hiatal hernia. It should be noted that Stretta is not available in some countries.

To date there have been four RCTs, 23 cohort studies, and two systematic reviews (one of which was a meta-analysis). The four RCTs included three that compared Stretta with sham therapy [48-50], and one that compared Stretta with PPI use [51]. Overall, the quality of evidence from the RCTs on the efficacy of the Stretta procedure is low, especially as the most important objective outcome parameters, such as acid exposure time, have often been omitted. No convincing evidence has been provided that Stretta normalizes acid exposure or LES pressure, but results from these RCTs converge to show some significant improvement in symptom burden and quality of life in the short term, although longer term data are still lacking.

The meta-analysis performed by Fass et al. included both RCTs and cohort studies [52]. They concluded that Stretta is efficacious in improving both objective and subjective clinical end points, except basal LES pressure. Lipka et al. published a systematic review that was limited to the four RCTs [53]. The pooled results showed no difference between Stretta and sham or management with PPIs in patients with GERD for the outcomes of mean percentage time the $\mathrm{pH}$ was less than 4 over a 24-hour time course, LES pressure, ability to stop PPIs, or health-related quality of life.

In terms of the comparison of Stretta vs. fundoplication, two non-randomized prospective comparative studies have been published, but the methodology of these studies was flawed (with selection criteria differing in the two groups) and the definitions of end points and symptom measurements were heterogeneous $[54,55]$. However, some improvement in symptom scores was observed in both studies, while another prospective study of Stretta as rescue therapy after failed laparoscopic fundoplication also proved useful in a subset of patients [56].

\subsection{Antireflux mucosectomy (ARMS)}

In antireflux mucosectomy (ARMS), an endoscopic mucosal resection (EMR) is performed at the level of the cardia over 180 $270^{\circ}$ degrees of the circumference. The concept behind ARMS is based on observations of the scars that result after ESD or EMR of gastric lesions. In this case, the scarring resulting from the healing of the mucosal resection at the level of the cardia leads to a narrowing of the EGJ and changes the angle of His, thereby potentially reducing gastroesophageal reflux.

\section{RECOMMENDATION}

ESGE recommends against the use of antireflux mucosectomy (ARMS) in routine clinical practice in the treatment of GERD because of the lack of data and its potential complications.

Strong recommendation, low quality evidence, level of agreement $100 \%$.

Only three case series (single-arm interventional studies) have been reported including in total $39 \mathrm{PPI}$-refractory GERD patients without a sliding hernia or with a hernia no bigger than $2 \mathrm{~cm}$ [57-59]. A clinical response was achieved in 69\%$80 \%$ of patients, with dysphagia occurring in $13 \%$. Patient numbers are too small to draw any conclusions on safety and efficacy, and controlled data are lacking.

\section{Intractable constipation}

Constipation is a common clinical condition. It is generally treated with dietary measures, lifestyle modifications, (osmotic) laxatives, or a combination thereof. In patients with intractable symptoms, retrograde or antegrade lavage can be considered.

Percutaneous endoscopic cecostomy tube placement describes a technique of placing tubes in the colon. The general technique is comparable to percutaneous endoscopic gastrostomy tube placement; however, as the tube is placed directly into the colon, the risk of complications is generally higher and a significant mortality is noted. The most common indication for percutaneous endoscopic cecostomy tube placement is relief of colonic obstruction or antegrade irrigation for colonic motility disorders [60]. In general, studies on percutaneous endoscopic cecostomy are rare and data collection is retrospective.

Therefore, the aim within this ESGE guideline is for the first time to provide guidance on the technique and management of percutaneous endoscopic cecostomy tube placement. 


\subsection{Indications for percutaneous endoscopic cecostomy}

\section{RECOMMENDATION}

ESGE recommends endoscopic cecostomy only after conservative management with medical therapies or retrograde lavage has failed.

Strong recommendation, low quality evidence, level of agreement $93.3 \%$.

\subsection{Techniques for endoscopic cecostomy}

\section{RECOMMENDATION}

ESGE recommends fixing the cecum to the abdominal wall at three points (using T-anchors, a double-needle suturing device, or laparoscopic fixation) to prevent leaks and infectious adverse events, whatever percutaneous endoscopic cecostomy method is used.

Strong recommendation, very low quality evidence, level of agreement $86.7 \%$.

Three main techniques of percutaneous endoscopic cecostomy have been used in clinical practice [61,63,66-69]: the pull-through method, the "introducer" method, and laparoscopically assisted percutaneous endoscopic cecostomy (LAPEC). The limited data do not provide evidence as to which method should be preferred. As cecostomy is accompanied by a relatively high frequency of adverse events, which may be serious (especially if no fixation of the cecum is used), the procedure should be reserved for patients with otherwise intractable constipation without any other therapeutic option. The necessary steps for percutaneous endoscopic cecostomy include: good bowel cleansing, use of sedation, disinfection of the abdominal wall, transillumination, and fixation of the cecum to the abdominal wall. In procedures where metal anchors are used, these should be removed within 3-4 weeks of percutaneous endoscopic cecostomy tube placement.

\subsubsection{Pull-through method}

For the pull-through method $[63,69]$, a colonoscopy is first performed to identify a site for insertion. The point of maximal transillumination is infiltrated with local anesthetic and the cecum is fixed at three points under endoscopic control. An $18 \mathrm{G}$ Seldinger needle (in children, 12G) is then passed through the abdominal wall at the center of the sutured triangle and a guidewire is passed through the needle and grasped by a snare. The guidewire is withdrawn from the anus and a tube $(14-20 \mathrm{Fr}$ in adults; $12 \mathrm{Fr}$ in children) is attached to it and pulled through the abdominal wall. The final position of the internal bolster is checked endoscopically and the tube is attached to the abdominal wall by an external bolster.

\subsubsection{Introducer method}

For the introducer method $[61,66]$, after a site for puncture and fixation of the cecum has been identified, a small incision or a puncture is made and, using a Seldinger technique, an introducer is advanced into the cecum, before a definitive catheter is placed and fixed. Chait Trapdoor percutaneous cecostomy catheters ("multiple pigtails") or balloon catheters $(11-15 \mathrm{Fr})$ are the most frequently used with this technique. Chait Trapdoor cecostomy catheters may have several advantages: no balloon rupture (and subsequent leak) can occur, no buried bumper syndrome can occur, granulation tissue over- 
growth occurs less frequently, and these catheters are easily exchangeable.

\subsubsection{Laparoscopically assisted percutaneous endoscopic cecostomy (LAPEC)}

For LAPEC $[67,68]$, a colonoscopy is first performed to identify the cecum. Fixation is then performed laparoscopically or under laparoscopic control. A cecostomy tube is placed laparoscopically. Although the procedure can be performed with a single laparoscopic port for the camera, several centers add two extra ports to allow the cecum to be held to facilitate needle insertion and for suturing of the cecum to the abdominal wall.

\subsection{Choice of technique for endoscopic cecostomy}

\section{RECOMMENDATION}

ESGE suggests the endoscopic route in critically ill patients in whom cecostomy is considered.

ESGE suggests laparoscopically assisted percutaneous endoscopic cecostomy (LAPEC) as the preferred technique for patients whose clinical condition is good. Weak recommendation, very low quality evidence, level of agreement $86.7 \%$.

A direct comparison between purely endoscopic cecostomy and LAPEC is not available from the literature. The technical success rates of purely endoscopic cecostomy are greater than $80 \%$; complications occur in $30 \%-40 \%$ of patients, and quality of life improves in general for most patients, although acceptance is reduced in about $25 \%$ of patients, mostly because of pain [61-64,70-75]. While most complications are minor, deaths have been reported secondary to endoscopic cecostomy-induced (fecal) peritonitis. LAPEC shows a success rate of $95 \%$, which is higher than the technical success rate reported for endoscopic cecostomy [67]. In critically ill patients, however, the endoscopic route might be preferred in order to avoid surgery and extensive sedation.

\subsection{Colostomy at other locations}

\section{RECOMMENDATION}

ESGE recommends performing endoscopic colostomy at no locations other than the cecum unless this is technically not feasible.

Strong recommendation, very low quality evidence, level of agreement $93.3 \%$.

Although data show the feasibility of performing colostomy at locations other than the cecum (left colon - descending or sigmoid) for patients suffering from constipation, there are no data demonstrating any advantage of this approach $[69,74,76$, 77]. Importantly, in some studies, there was a very high and unacceptable incidence of serious adverse events $[69,74]$. In one retrospective study, analyzing 31 patients who underwent endoscopic colostomy on the left side of the colon, recurrent complications and infection caused significant morbidity and necessitated percutaneous endoscopic colostomy tube removal in most patients ( 13 of the 14 patients with constipation had to have the tube removed). Moreover, two patients died because of fecal peritonitis [74].

\section{Ogilvie's syndrome}

Ogilvie's syndrome, also known as acute colonic pseudoobstruction, refers to pathologic dilatation of the colon without underlying mechanical obstruction [78]. It occurs primarily in patients with serious comorbidities.

In patients with signs or symptoms of acute colonic dilatation, the presence of mechanical large-bowel obstruction should be excluded with an abdominal computed tomography (CT) scan or water-soluble contrast enema. Furthermore, routine blood testing, including complete blood count, serum electrolytes, renal function assessment, and thyroid function, should be performed during the initial evaluation to check for predisposing and potentially correctable factors (i.e. electrolyte imbalance, renal insufficiency, infection, and hypothyroidism).

\subsection{Indications for endoscopic treatment}

\subsubsection{Endoscopic decompression vs. neostigmine}

\section{RECOMMENDATION}

ESGE recommends considering endoscopic decompression of the colon in patients with Ogilvie's syndrome that is not improving with conservative treatment.

Strong recommendation, low quality evidence, level of agreement $93.8 \%$.

Conservative therapy is the initial step in the management of patients with Ogilvie's syndrome. In the current literature, the following actions have been described: discontinuation of narcotics, anticholinergics, and calcium-channel antagonists; correction of electrolyte abnormalities; nil per os; decompressing the GI tract by nasogastric tube and/or rectal tube insertion, and frequent position changes [79]. Because these recommendations have never been studied as a single intervention, their effects are unknown.

In patients with Ogilvie's syndrome that is not improving with conservative treatment, both endoscopic decompression therapy and medical therapy with intravenous neostigmine are considered valid treatment options. The efficacy of endoscopic decompression was investigated in several retrospective studies, in which the overall success rates varied between $36 \%$ and $88 \%$ [79-81].

In a recent study, Peker et al. demonstrated that, compared with neostigmine, endoscopic decompression was more effective as an initial therapy and was more effective at avoiding a 
second treatment modality (total response $82 \%$ vs. $49 \%$, $P<0.001$ ) [80]. Comparable results were shown in the study of Tsirline et al. in which colonoscopy was significantly more successful than single or repeated neostigmine administration (no further therapy after one or two interventions: $75.0 \%$ vs. $35.5 \%, P<0.001$; and $84.6 \%$ vs. $55.6 \%, P=0.003$, respectively) [81]. However, in these two retrospective studies, the efficacy of neostigmine treatment was much lower compared with previous studies, in which the efficacy of neostigmine ranged between $61 \%$ and $100 \%[79,82-84]$.

Regarding safety, the risk of perforation due to endoscopic decompression is described in $0-5 \%$ of patients [79]. However, perforations are also described in patients with Ogilvie's syndrome receiving conservative or neostigmine treatment [81]. Ross et al. demonstrated that patients who fail on medical management and require interventional procedures, including endoscopic decompression, experience increasing morbidity and mortality with increasing invasiveness of the procedure, likely reflecting the severity of their conditions [85].

Because no prospective head-to-head comparisons between endoscopic decompression and neostigmine treatment are available, no recommendation can be made for the superiority of one of these two treatment strategies in patients with Ogilvie's syndrome that is not improving with conservative treatment. Furthermore, there is a large heterogeneity regarding the patient population, definition of success, and treatment protocols, which makes a good comparison between studies difficult. The choice of treatment should also depend on local expertise and the local situation, for instance the access to urgent colonoscopy.

\subsubsection{Criteria for prompt endoscopic decompression}

\section{RECOMMENDATION}

ESGE recommends prompt endoscopic decompression if the cecal diameter is $>12 \mathrm{~cm}$ and if the Ogilvie's syndrome exists for a duration longer than 4-6 days.

Strong recommendation, low quality evidence, level of agreement $87.5 \%$.

The relationships between cecal diameter and duration of distension in patients with Ogilvie's syndrome and risk of perforation and ischemia were investigated in two retrospective studies. In one study, the risk of perforation and/or ischemia was higher with increasing cecal diameter: $<12 \mathrm{~cm}, 0 \%(\mathrm{n}=44)$; $12-14 \mathrm{~cm}, 7 \%(\mathrm{n}=29) ;>14 \mathrm{~cm}, 23 \%(\mathrm{n}=69)$. A cecal diameter $>14 \mathrm{~cm}$ was associated with a two-fold increase in mortality. In addition, a delay in decompression was associated with higher mortality: <4 days, 15\%; 4-7 days, 27\%; >7 days, 73\% [86]. Johnson et al. demonstrated that the risk of perforation was related more to duration of cecal distension ( $>6$ days) than to the absolute cecal size [87].

When colonic ischemia and/or perforation occur, patients are no longer considered eligible for endoscopic management and should be referred for surgery.

\subsubsection{Recurrence of Ogilvie’s syndrome}

\section{RECOMMENDATION}

ESGE recommends considering repeated endoscopic decompression for recurrence of Ogilvie's syndrome.

Strong recommendation, low quality evidence, level of agreement $92.9 \%$.

The risk of recurrence of Ogilvie's syndrome after an initial successful decompression varies widely in the current literature, ranging from $0-38 \%$ in patients previously treated with neostigmine and $0-50 \%$ after endoscopic decompression [88].

Repeated endoscopic decompression for recurrence of Ogilvie's syndrome may still be effective. Some studies report similar success rates of a second or third colonic decompression compared with the initial decompression, although the sample sizes were small $[81,89]$. Other studies suggest that repeated endoscopic decompression is associated with lower, but still acceptable, sustained clinical success rates: Geller et al. showed that clinical success was achieved in a significantly higher percentage of patients undergoing a single decompression compared with those requiring multiple procedures (95\% vs. $56 \%$, $P<0.05$ ) [88]. In the study of Vanek et al., repeat colonoscopic decompression demonstrated an $87 \%$ success rate, comparable with the initial colonoscopies, but a higher recurrence rate of $40 \%$ vs. $22 \%$ after the initial colonoscopies [86].

As for the initial treatment for Ogilvie's syndrome that is not responding to conservative management, no prospective headto-head comparisons between endoscopic decompression and neostigmine treatment are available for recurrent Ogilvie's syndrome, making the choice of treatment dependent on local expertise and the local situation, such as the access to urgent colonoscopy.

\subsection{Periprocedural management of endoscopic decompression}

\subsubsection{Use of a decompression tube}

\section{RECOMMENDATION}

ESGE recommends the placement of a decompression tube in the right or transverse colon after endoscopic decompression as this seems to be associated with lower recurrence rates.

Strong recommendation, low quality evidence, level of agreement $92.9 \%$.

Several old retrospective studies investigated the use of decompression tube placement after endoscopic decompression in patients with Ogilvie's syndrome. Geller et al. showed in a study comprising 50 patients that the rate of clinical success, defined as a sustained decompression, was $80 \%$ in 
patients with endoscopic decompression tube placement vs. $25 \%$ without tube placement [88]. Placement of a decompression tube in the right or transverse colon had a similar effect ( $90 \%$ vs. $83 \%, P>0.05$ ); however, the success rate was lower after tube placement in the hepatic flexure (63\%), splenic flexure $(75 \%)$, or descending colon (0\%) [88]. These findings are in line with those of smaller studies [90-92].

In conclusion, most data suggest lower recurrence rates in patients with decompression tube placement after endoscopic decompression. Tube placement in the right or transverse colon seems to have similar effects. However, these conclusions are based on relatively old and small retrospective studies and RCTs are missing. There is no information mentioned in the literature regarding the duration for which the decompression tube should be kept in place. In clinical practice, however, decompression tubes are kept in place for 1 - 3 days, and spontaneous expulsion of the tube before the intended time of removal is not a rare event. Low (intermittent) suction can be applied, and regular flushing (every $2-4$ hours) with $20-30 \mathrm{~mL}$ of normal saline is generally advised to maintain patency.

\subsubsection{Bowel preparation}

\section{RECOMMENDATION}

ESGE recommends against the use of oral bowel preparation solutions prior to colonic decompression as these may worsen dilatation of the colon.

Strong recommendation, very low quality evidence, level of agreement $93.8 \%$.

There are no studies regarding bowel preparation in patients with Ogilvie's syndrome undergoing endoscopic decompression. However, use of oral bowel preparation solutions is not recommended prior to colonic decompression as these may worsen dilatation of the colon in the absence of bowel transit. The usefulness of enemas before endoscopic decompression has never been investigated and remains unclear.

\subsubsection{Post-procedural oral PEG solution}

\section{RECOMMENDATION}

ESGE recommends the administration of oral PEG solution in patients with Ogilvie's syndrome after initial resolution of colonic dilatation as it decreases the risk of recurrence.

Strong recommendation, low level of evidence, level of agreement $100 \%$.

One small randomized placebo-controlled trial including 30 patients who initially responded to neostigmine or colonoscopic decompression demonstrated that administration of oral PEG solution significantly decreases the rate of relapse compared with placebo ( $0 \%$ vs. $33 \%, P=0.04$ ) [93]. The pre- scription of other types of laxatives after successful decompression, especially those exerting an effect on colonic motility, seems reasonable and rational, but supportive studies are lacking.

\subsection{Role of percutaneous endoscopic colostomy}

\section{RECOMMENDATION}

ESGE recommends considering percutaneous endoscopic colostomy/cecostomy for patients with Ogilvie's syndrome that is refractory to pharmacologic and endoscopic treatment, especially in those not amenable to surgical intervention because of an increased perioperative risk.

Strong recommendation, very low quality evidence, level of agreement $80.0 \%$.

Several studies report data regarding percutaneous endoscopic colostomy placement for various indications; however, the total number of patients with Ogilvie's syndrome is relatively low. Baraza et al. performed 35 percutaneous endoscopic colostomies in 33 patients, of whom four had recurrent Ogilvie's syndrome and were considered poor candidates for an operation [69]. Symptoms resolved in $74 \%$ of all patients, including in three of the four patients with recurrent Ogilvie's syndrome. Major complications occurred in four patients: three cases of peritonitis secondary to fecal contamination and one death. In another small study, eight percutaneous endoscopic cecostomies were performed: six for colonic pseudo-obstruction and two for chronic constipation [63], with seven of the eight cases successful and resulting in clinical improvement. One patient required surgical removal of the percutaneous endoscopic cecostomy tube for fecal spillage resulting in peritonitis. In a retrospective study from Cowlam et al., an improvement in symptoms was reported in $81 \%$ of 31 patients after a percutaneous endoscopic colostomy was performed, including in five patients with acute-on-chronic colonic pseudo-obstruction. One of these five patients died from fecal peritonitis; in three, the tube was removed because of infection [74]. Similar results were shown in other case reports $[71,94,95]$.

Percutaneous endoscopic colostomy, although certainly not devoid of complications, has two potential advantages over surgery in patients with refractory Ogilvie's syndrome. Most importantly, general anesthesia can be avoided. Furthermore, tube placement is reversible after an improvement in symptoms. Therefore, percutaneous endoscopic colostomy should be considered as an alternative to surgery in patients with Ogilvie's syndrome that is refractory to pharmacologic and endoscopic treatment, despite the fact that there are no comparative data.

\section{Disclaimer}

The legal disclaimer for ESGE guidelines [2] applies to this Guideline. 


\section{Acknowledgments}

The authors gratefully thank Dr. M. Pioche of the Hôpital Edouard Herriot, Lyon and Dr. T. Beyna of the Evangelisches Krankenhaus, Duesseldorf for their valuable comments on the manuscript.

Lastly, we would like to thank Claire Guy for arranging the Delphi procedure, and Pauline Rocheteau and Francoise Heidenreich for organizing all the meetings and telephone conferences for the Guideline.

\section{Competing interests}

A.J. Bredenoord has received speaker's fees from MMS, Diversatek, and Medtronics (ongoing). P. Familiari received speaker's fees from Olympus (October 2019). H. Neumann has provided consultancy services to Fujifilm, Pentax, Motus Gl, Boston Scientific, and Cook Medical (2012 to present). D. Pohl has provided consultancy services to Medtronic (2018 to present). R. Tutuian has provided consultancy services and educational programs to Laborie/MMS (2010 to present). J.E. van Hooft has received lecture fees from Medtronics (2014-2015) and consultancy fees from Boston Scientific (20142017); her department has received research grants from Cook Medical (2014-2018) and Abbott (2014-2017). D. von Renteln has received research funding from Pendopharm (2016-2019), Ventage and Pentax (2018 - 2019), ERBE (2019 to present), and Boston Scientific (2020), and speaker's fees from Boston Scientific (2018-2020) and ERBE (2020). M. Barret, J.-M. Gonzalez, S. Ishaq, V. LorenzoZúñiga, H. Louis, J. Martinek, F. Prat, E. Savarino, R. Sweis, J. Tack, S. van Meer, and B.L.A.M. Weusten declare that they have no conflict of interest.

\section{References}

[1] Atkins D, Best D, Briss P et al. Grading quality of evidence and strength of recommendations. BMJ 2004; 328: 1490

[2] Dumonceau JM, Hassan C, Riphaus A et al. European Society of Gastrointestinal Endoscopy (ESGE) guideline development policy. Endoscopy 2012; 44: 626-629

[3] Linstone HA, Turoff M. The Delphi Method: Techniques and Applications. 2002: Available from: https://web.njit.edu/ turoff/pubs/delphibook/index.html

[4] Watemberg S, Landau O, Avrahami R. Zenker's diverticulum: reappraisal. Am J Gastroenterol 1996; 91: 1494-1498

[5] Wheeler WI. Pharyngocele and dilatation of pharynx, with existing diverticulum at lower poertion of pharynx lying posterior to the oesophagus, cured by pharyngotomy, being the first case of the kind recorded. Dublin J Med Sci 1886; 82: 349-357

[6] Negus V. Pharyngeal diverticula: observations on their evolution and treatment. Br J Surg 1950; 38: 129-146

[7] Fulp S, Castell D. Manometric aspects of Zenker's diverticulum. Hepatogastroenterology 1992; 39: 123-126

[8] Cook I, Gabb M, Panagopoulos V et al. Pharyngeal (Zenker's) diverticulum is a disorder of the upper esophageal sphincter opening. Gastroenterology 1992; 103: 1229-1235

[9] Mantsopoulos K, Psychogios G, Künzel J et al. Evaluation of the different transcervical approaches for Zenker diverticulum. Otolaryngol Head Neck Surg 2012; 146: 725-729

[10] Yuan Y, Zhao Y, Hu Y et al. Surgical treatment of Zenker's diverticulum. Dig Surg 2013; 30: 207-218
[11] Leong S, Wilkie M, Webb C. Endoscopic stapling of Zenker's diverticulum: Establishing national baselines for auditing clinical outcomes in the United Kingdom. Eur Arch Otorhinolaryngol 2012; 269: 18771884

[12] Luna R, Collard J. Transoral stapled diverticulotomy. Rev Col Bras Cir 2009; 36: 268-270

[13] Mulder C], den Hartog G, Robijn RJ et al. Flexible endoscopic treatment of Zenker's diverticulum: a new approach. Endoscopy 1995; 27: 438-442

[14] Huberty V, El Bacha S, Blero D et al. Endoscopic treatment for Zenker's diverticulum: Long-term results (with video). Gastrointest Endosc 2013; 77: 701-707

[15] Antonello A, Ishaq S, Zanatta L et al. The role of flexible endotherapy for the treatment of recurrent Zenker's diverticula after surgery and endoscopic stapling. Surg Endosc 2016; 30: 2351-2357

[16] Shahawy S, Janisiewicz AM, Annino D et al. A comparative study of outcomes for endoscopic diverticulotomy versus external diverticulectomy. Otolaryngol Head Neck Surg 2014; 151: 646-651

[17] Verdonck J, Morton RP. Systematic review on treatment of Zenker's diverticulum. Eur Arch Otorhinolaryngol 2015; 272: 3095-3107

[18] Albers D, Kondo A, Bernardo W et al. Endoscopic versus surgical approach in the treatment of Zenker's diverticulum: systematic review and meta-analysis. Endosc Int Open 2016; 04: E678-E686

[19] Ishaq S, Hassan C, Antonello A et al. Flexible endoscopic treatment for Zenker's diverticulum: A systematic review and meta-analysis. Gastrointest Endosc 2016; 83: 1076-1089.e5

[20] Costamagna G, lacopini F, Bizzotto A et al. Prognostic variables for the clinical success of flexible endoscopic septotomy of Zenker's diverticulum. Gastrointest Endosc 2016; 83: 765-773

[21] Oestreicher-Kedem Y, Wasserzug O, Sagi B et al. Revision endoscopic stapler Zenker's diverticulotomy. Surg Endosc 2016; 30: 2022-2025

[22] Buchanan MA, Riffat F, Mahrous AK et al. Endoscopic or external approach revision surgery for pharyngeal pouch following primary endoscopic stapling: Which is the favoured approach? Eur Arch Otorhinolaryngol 2013; 270: 1707-1710

[23] Costamagna G, lacopini F, Tringali A et al. Flexible endoscopic Zenker's diverticulotomy: cap-assisted technique vs. diverticuloscopeassisted technique. Endoscopy 2007; 39: 146-152

[24] Yang J, Novak S, Ujiki M et al. An international study on the use of peroral endoscopic myotomy in the management of Zenker's diverticulum. Gastrointest Endosc 2020; 91: 163-168

[25] Ishioka S, Sakai P, Maluf Filho F et al. Endoscopic incision of Zenker's diverticula. Endoscopy 1995; 27: 433-437

[26] Repici A, Pagano F, Danese $S$ et al. Endoscopic flexible treatment of Zenker's diverticulum: a modification of the needle-knife technique. Endoscopy 2010; 42: 532-535

[27] Rouquette $O$, Abergel A, Mulliez A et al. Usefulness of the Hook knife in flexible endoscopic myotomy for Zenker's diverticulum. World ] Gastrointest Endosc 2017; 9: 411-416

[28] Battaglia G, Antonello A, Realdon S et al. Flexible endoscopic treatment for Zenker's diverticulum with the SB Knife. Preliminary results from a single-center experience. Dig Endosc 2015; 27: 728-733

[29] Gölder SK, Brueckner J, Ebigbo A et al. Double incision and snare resection in symptomatic Zenker's diverticulum: A modification of the stag beetle knife technique. Endoscopy 2018; 50: 137-141

[30] El-Serag HB, Sweet S, Winchester CC et al. Update on the epidemiology of gastro-oesophageal reflux disease: A systematic review. Gut 2014; 63: 871-880

[31] Cohen LB, Johnson DA, Ganz RA et al. Enteryx implantation for GERD: Expanded multicenter trial results and interim postapproval follow-up to 24 months. Gastrointest Endosc 2005; 61: 650-658 
[32] Wong RF, Davis TV, Peterson KA. Complications involving the mediastinum after injection of Enteryx for GERD. Gastrointest Endosc 2005; 61: 753-756

[33] Fockens P, Bruno M], Gabbrielli A et al. Endoscopic augmentation of the lower esophageal sphincter for the treatment of gastroesophageal reflux disease: multicenter study of the Gatekeeper Reflux Repair System. Endoscopy 2004; 36: 682-689

[34] Fockens P, Cohen L, Edmundowicz SA et al. Prospective randomized controlled trial of an injectable esophageal prosthesis versus a sham procedure for endoscopic treatment of gastroesophageal reflux disease. Surg Endosc 2010; 24: 1387-1397

[35] Mahmood Z, McMahon BP, Arfin Q et al. Endocinch therapy for gastrooesophageal reflux disease: A one year prospective follow up. Gut 2003; 52: 34-39

[36] Schiefke I, Zabel-Langhennig A, Neumann S et al. Long term failure of endoscopic gastroplication (EndoCinch). Gut 2005; 54: 752-758

[37] Siersema PD. Endoscopic therapeutic esophageal interventions: what is new? What needs further study? What can we forget? Curr Opin Gastroenterol 2005; 21: 490-497

[38] Rinsma NF, Farré R, Bouvy ND et al. The effect of endoscopic fundoplication and proton pump inhibitors on baseline impedance and heartburn severity in GERD patients. Neurogastroenterol Motil 2015; 27: $220-228$

[39] Witteman BPL, Conchillo JM, Rinsma NF et al. Randomized controlled trial of transoral incisionless fundoplication vs. proton pump inhibitors for treatment of gastroesophageal reflux disease. Am J Gastroenterol 2015; 110: 531-542

[40] Trad KS, Barnes WE, Simoni G et al. Transoral incisionless fundoplication effective in eliminating GERD symptoms in partial responders to proton pump inhibitor therapy at 6 months: The TEMPO randomized clinical trial. Surg Innov 2015; 22: 26-40

[41] Hunter JG, Kahrilas PJ, Bell RCW et al. Efficacy of transoral fundoplication vs omeprazole for treatment of regurgitation in a randomized controlled trial. Gastroenterology 2015; 148: 324-333

[42] Håkansson B, Montgomery M, Cadiere GB et al. Randomised clinical trial: Transoral incisionless fundoplication vs. sham intervention to control chronic GERD. Aliment Pharmacol Ther 2015; 42: 1261-1270

[43] Huang X, Chen S, Zhao $\mathrm{H}$ et al. Efficacy of transoral incisionless fundoplication (TIF) for the treatment of GERD: a systematic review with meta-analysis. Surg Endosc 2017; 31: 1032-1044

[44] Frazzoni M, Conigliaro R, Manta R et al. Reflux parameters as modified by EsophyX or laparoscopic fundoplication in refractory GERD. Aliment Pharmacol Ther 2011; 34: 67-75

[45] Zacherl J, Roy-Shapira A, Bonavina L et al. Endoscopic anterior fundoplication with the Medigus Ultrasonic Surgical Endostapler (MUSE ${ }^{\mathrm{TM}}$ ) for gastroesophageal reflux disease: 6-month results from a multicenter prospective trial. Surg Endosc 2015; 29: 220-229

[46] Kim HJ, Kwon Cll, Kessler WR et al. Long-term follow-up results of endoscopic treatment of gastroesophageal reflux disease with the MUSETM endoscopic stapling device. Surg Endosc 2016; 30: 34023408

[47] Danalioglu A, Cipe G, Toydemir T et al. Endoscopic stapling in comparison to laparoscopic fundoplication for the treatment of gastroesophageal reflux disease. Dig Endosc 2014; 26: 37-42

[48] Arts ], Bisschops R, Blondeau K et al. A double-blind sham-controlled study of the effect of radiofrequency energy on symptoms and distensibility of the gastro-esophageal junction in GERD. Am J Gastroenterol 2012; 107: 222-230

[49] Corley DA, Katz P, Wo JM et al. Improvement of gastroesophageal reflux symptoms after radiofrequency energy: A randomized, shamcontrolled trial. Gastroenterology 2003; 125: 668-676
[50] Abdel Aziz AM, El-Khayat HR, Sadek A et al. A prospective randomized trial of sham, single-dose Stretta, and double-dose Stretta for the treatment of gastroesophageal reflux disease. Surg Endosc 2010; 24: 818-825

[51] Coron E, Sebille V, Cadiot G et al. Clinical trial: Radiofrequency energy delivery in proton pump inhibitor-dependent gastro-oesophageal reflux disease patients. Aliment Pharmacol Ther 2008; 28: 11471158

[52] Fass R, Cahn F, Scotti D] et al. Systematic review and meta-analysis of controlled and prospective cohort efficacy studies of endoscopic radiofrequency for treatment of gastroesophageal reflux disease. Surg Endosc 2017; 31: 4865-4882

[53] Lipka S, Kumar A, Richter JE. No evidence for efficacy of radiofrequency ablation for treatment of gastroesophageal reflux disease: a systematic review and meta-analysis. Clin Gastroenterol Hepatol 2015; 13: 1058-1067

[54] Richards WO, Houston HL, Torquati A et al. Paradigm shift in the management of gastroesophageal reflux disease. Ann Surg 2003; 237: 638-649

[55] Liang W-T, Yan C, Wang Z-G et al. Early and midterm outcome after laparoscopic fundoplication and a minimally invasive endoscopic procedure in patients with gastroesophageal reflux disease: a prospective observational study. J Laparoendosc Adv Surg Tech 2015; 25 : 657-661

[56] Noar M, Squires P, Khan S. Radiofrequency energy delivery to the lower esophageal sphincter improves gastroesophageal reflux patient-reported outcomes in failed laparoscopic Nissen fundoplication cohort. Surg Endosc 2017; 31: 2854-2862

[57] Inoue $\mathrm{H}$, Ito $\mathrm{H}$, Ikeda $\mathrm{H}$ et al. Anti-reflux mucosectomy for gastroesophageal reflux disease in the absence of hiatus hernia: A pilot study. Ann Gastroenterol 2014; 27: 346-351

[58] Benias P, D'Souza L, Lan G et al. Initial experience with a novel resection and plication (RAP) method for acid reflux: a pilot study. Endosc Int Open 2018; 06: E443-E449

[59] Hedberg HM, Kuchta K, Ujiki MB. First experience with banded antireflux mucosectomy (ARMS) for GERD: feasibility, safety, and technique (with video). J Gastrointest Surg 2019; 23: 1274-1278

[60] Holm AN, Baron TH. Palliative use of percutaneous endoscopic gastrostomy and percutaneous endoscopic cecostomy tubes. Gastrointest Endosc Clin N Am 2007; 17: 795-803

[61] Duchalais E, Meurette G, Mantoo SK et al. Percutaneous endoscopic caecostomy for severe constipation in adults: feasibility, durability, functional and quality of life results at 1 year follow-up. Surg Endosc 2015; 29: 620-626

[62] Küllmer A, Schmidt A, Caca K. Percutaneous endoscopic cecostomy (introducer method) in chronic intestinal pseudo-obstruction: Report of two cases and literature review. Dig Endosc 2016; 28: 210-215

[63] Lynch CR, Jones RG, Hilden K et al. Percutaneous endoscopic cecostomy in adults: a case series. Gastrointest Endosc 2006; 64: 279-282

[64] Ricard J, Quénéhervé L, Lefevre C et al. Anterograde colonic irrigations by percutaneous endoscopic caecostomy in refractory colorectal functional disorders. Int J Colorectal Dis 2019; 34: 169-175

[65] Didailler R, Denost Q, Loughlin P et al. Antegrade enema after total mesorectal excision for rectal cancer: the last chance to avoid definitive colostomy for refractory low anterior resection syndrome and fecal incontinence. Dis Colon Rectum 2018; 61: 667-672

[66] Uno Y. Introducer method of percutaneous endoscopic cecostomy and antegrade continence enema by use of the Chait Trapdoor cecostomy catheter in patients with adult neurogenic bowel. Gastrointest Endosc 2006; 63: 666-673

[67] Koyfman S, Swartz K, Goldstein AM et al. Laparoscopic-assisted percutaneous endoscopic cecostomy (LAPEC) in children and young adults. J Gastrointest Surg 2017; 21: 676-683 
[68] Rodriguez L, Flores A, Gilchrist BF et al. Laparoscopic-assisted percutaneous endoscopic cecostomy in children with defecation disorders (with video). Gastrointest Endosc 2011; 73: 98-102

[69] Baraza W, Brown S, McAlindon M et al. Prospective analysis of percutaneous endoscopic colostomy at a tertiary referral centre. $\mathrm{Br}$ J Surg 2007; 94: 1415-1420

[70] Rivera MT, Kugathasan S, Berger W et al. Percutaneous colonoscopic cecostomy for management of chronic constipation in children. Gastrointest Endosc 2001; 53: 225-228

[71] Ramage JI, Baron TH. Percutaneous endoscopic cecostomy: a case series. Gastrointest Endosc 2003; 57: 752-755 3

[72] Cascio S, Flett ME, De La Hunt M et al. MACE or caecostomy button for idiopathic constipation in children: A comparison of complications and outcomes. Pediatr Surg Int 2004; 20: 484-487

[73] Biyani D, Barrow E, Hodson P et al. Endoscopically placed caecostomy buttons: A trial ACE procedure. Colorectal Dis 2007; 9: 373-376

[74] Cowlam S, Watson C, Elltringham M et al. Percutaneous endoscopic colostomy of the left side of the colon. Gastrointest Endosc 2007; 65: 1007-1014

[75] Graham CD, Rodriguez L, Flores A et al. Primary placement of a skinlevel cecostomy tube for antegrade colonic enema administration using a modification of the laparoscopic-assisted percutaneous endoscopic cecostomy (LAPEC). J Pediatr Surg 2019; 54: 486-490

[76] Rawat DJ, Haddad M, Geoghegan N et al. Percutaneous endoscopic colostomy of the left colon: A new technique for management of intractable constipation in children. Gastrointest Endosc 2004; 60: 39-43

[77] Rao AS, Loftus CG, Baron TH. Buried bumper syndrome arising from a percutaneous endoscopic cecostomy tube. Gastrointest Endosc 2011; 73: 168-169

[78] Ogilvie H. Large-intestine colic due to sympathetic deprivation; a new clinical syndrome. Br Med J 1948; 2: 671-673

[79] Saunders MD. Acute colonic pseudo-obstruction. Best Pract Res Clin Gastroenterol 2007; 21: 671-687

[80] Peker KD, Cikot M, Bozkurt MA et al. Colonoscopic decompression should be used before neostigmine in the treatment of Ogilvie's syndrome. Eur J Trauma Emerg Surg 2017; 43: 557-566

[81] Tsirline VB, Zemlyak AY, Avery MJ et al. Colonoscopy is superior to neostigmine in the treatment of Ogilvie's syndrome. Am J Surg 2012; 204: 849-855
[82] Ponec R], Saunders MD, Kimmey MB. Neostigmine for the treatment of acute colonic pseudo-obstruction. NEJM 1999; 341: 137-141

[83] Elsner JL, Smith JM, Ensor CR. Intravenous neostigmine for postoperative acute colonic pseudo-obstruction. Ann Pharmacother 2012; 46: 430-435

[84] Valle RGL, Godoy FL. Neostigmine for acute colonic pseudo-obstruction: A meta-analysis. Ann Med Surg 2014; 3: 60-64

[85] Ross SW, Oommen B, Wormer BA et al. Acute colonic pseudo-obstruction: Defining the epidemiology, treatment, and adverse outcomes of Ogilvie's syndrome. Am Surg 2016; 82: 102-111

[86] Vanek VW, Al-Salti M. Acute pseudo-obstruction of the colon (Ogilvie's syndrome). Dis Colon Rectum 1986; 29: 203-210

[87] Johnson CD, Rice RP, Kelvin FM et al. The radiologic evaluation of gross cecal distension: Emphasis of cecal ileus. Am J Roentgenol 1985; 145: 1211-1217

[88] Geller A, Petersen BT, Gostout C]. Endoscopic decompression for acute colonic pseudo-obstruction. Gastrointest Endosc 1996; 44: 144-150

[89] Jetmore AB, Timmcke AE, Gathright JB et al. Ogilvie's syndrome: Colonoscopic decompression and analysis of predisposing factors. Dis Colon Rectum 1992; 35: 1135-1142

[90] Harig JM, Fumo DE, Loo FD et al. Treatment of acute nontoxic megacolon during colonoscopy: tube placement versus simple decompression. Gastrointest Endosc 1988; 34: 23-27

[91] Lavignolle A, Jutel P, Bonhomme J et al. Ogilvie's syndrome: results of endoscopic exsufflation in a series of 29 cases. Gastroenterol Clin Biol 1986; 10: 147-151

[92] Pham TN, Cosman BC, Chu P et al. Radiographic changes after colonoscopic decompression for acute pseudo-obstruction. Dis Colon Rectum 1999; 42: 1586-1591

[93] Sgouros SN, Vlachogiannakos ], Vassiliadis K et al. Effect of polyethylene glycol electrolyte balanced solution on patients with acute colonic pseudo obstruction after resolution of colonic dilation: A prospective, randomised, placebo controlled trial. Gut 2006; 55: 638642

[94] Ponsky JL, Aszodi A, Perse D. Percutaneous endoscopic cecostomy: a new approach to nonobstructive colonic dilation. Gastrointest Endosc 1986; 32: 108-111

[95] Salm R, Rückauer K, Waldmann D et al. Endoscopic percutaneous cecostomy (EPC). Surg Endosc 1988; 2: 92-95

\section{CORRECTION}

Endoscopic management of gastrointestinal motility disorders - part 2: European Society of Gastrointestinal Endoscopy (ESGE) Guideline

Weusten BLA.M., Barret M, Bredenoord AJ et al. Endoscopy 2020; 52: 600-614

In the above-mentioned article, the institution of Daniel Pohl has been corrected.

This was corrected in the online version on June 24, 2020. 